\title{
Clinical Study \\ Bowel Preparation for Colonoscopy with Sodium Phosphate Solution versus Polyethylene Glycol-Based Lavage: A Multicenter Trial
}

\author{
S. Schanz, ${ }^{1}$ W. Kruis, ${ }^{1}$ O. Mickisch, ${ }^{2}$ B. Küppers, ${ }^{2}$ P. Berg, ${ }^{3}$ B. Frick, ${ }^{3}$ G. Heiland, ${ }^{3}$ D. Hüppe, ${ }^{4}$ \\ B. Schenck, ${ }^{4}$ H. Horstkotte, ${ }^{5}$ and A. Winkler ${ }^{5}$ \\ ${ }^{1}$ Department of Internal Medicine and Gastroenterology, Evangelic Hospital Kalk, Buchforststrasse 2, \\ 51103 Koeln, Germany \\ ${ }^{2}$ Gastroenterologische Gemeinschaftspraxis, O7, Nr 14 (Am Wasserturm), 68161 Mannheim, Germany \\ ${ }^{3}$ Gastroenterologische Gemeinschaftspraxis, Dieburger Strasse 29, 64287 Darmstadt, Germany \\ ${ }^{4}$ Gastroenterologische Gemeinschaftspraxis, Wiescherstrasse 20, 44623 Herne, Germany \\ ${ }^{5}$ Gastroenterologische Gemeinschaftspraxis, Schmiedestrasse 18, 30159 Hannover, Germany
}

Correspondence should be addressed to S. Schanz, stefan.schanz@t-online.de

Received 30 October 2007; Revised 19 March 2008; Accepted 8 June 2008

Recommended by Guenter Janetschek

Background: Adequate bowel preparation is essential for accurate colonoscopy. Both oral sodium phosphate (NaP) and polyethylene glycol-based lavage (PEG-ELS) are used predominantly as bowel cleansing modalities. NaP has gained popularity due to low drinking volume and lower costs. The purpose of this randomized multicenter observer blinded study was to compare three groups of cleansing ( $\mathrm{NaP}, \mathrm{NaP}+$ sennosides, PEG-ELS + sennosides) in reference to tolerability, acceptance, and cleanliness. Patient and Methods: 355 outpatients between 18 and 75 years were randomized into three groups $(\mathrm{A}, \mathrm{B}, \mathrm{C})$ receiving $\mathrm{NaP}=$ $\mathrm{A}, \mathrm{NaP}$, and sennosides = B or PEG-ELS and sennosides $=\mathrm{C}$. Gastroenterologists performing colonoscopies were blinded to the type of preparation. All patients documented tolerance and adverse events. Vital signs, premedication, completeness, discomfort, and complications were recorded. A quality score (0-4) of cleanliness was generated. Results: The three groups were similar with regard to age, sex, BMI, indication for colonoscopy, and comorbidity. Drinking volumes $(\mathrm{L})(\mathrm{A}=4.33+1.2, \mathrm{~B}=4.56+1.18, \mathrm{C}$ $=4.93+1.71)$ were in favor of $\mathrm{NaP}(P=.005)$. Discomfort from ingested fluid was recorded in $\mathrm{A}=39.8 \%$ (versus $\mathrm{C}: P=.015)$, $\mathrm{B}$ $=46.6 \%$ (versus C: $P=.147$ ), and C $=54.6 \%$. Differences in tolerability and acceptance between the three groups were statistically not significant. No differences in adverse events and the cleanliness effects occurred in the three groups $(P=.113)$. The cleanliness quality scores 0-2 were calculated in A: 77.7\%, B: $86.7 \%$, and C: $85.2 \%$. Conclusions: These data fail to demonstrate significant differences in tolerability, acceptance, and preparation quality between the three types of bowel preparation for colonoscopy. Cleansing with $\mathrm{NaP}$ was not superior to PEG-ELS.

Copyright (c) 2008 S. Schanz et al. This is an open access article distributed under the Creative Commons Attribution License, which permits unrestricted use, distribution, and reproduction in any medium, provided the original work is properly cited.

\section{INTRODUCTION}

Colonoscopy is performed as a principal diagnostic tool in most colonic disorders. Adequate bowel preparation is essential for proper visualization of mucosa. Superior cleansing minimizes the risks of missed lesions and repeated procedures, and decreases patients' discomfort and costs of colonoscopy $[1,2]$.

Peroral polyethylene glycol (PEG) solution has been the cleansing agent most used in recent years. This nondigestible, nonabsorbable osmotically balanced laxative lavage solution does not alter fluid and electrolyte balance [3-6]. Despite the improved tolerance to the two-day standard preparation of clear liquids, laxatives, and enemas, the large fluid intake may lead to nausea and abdominal discomfort.

Sodium phosphate $(\mathrm{NaP})$, a highly osmotic laxative, has also proven to be an effective and well-tolerated agent [7]. Because of the small quantity needed, it has become one of the preferred agents. Over the past decade, numerous studies have demonstrated that oral $\mathrm{NaP}$ is even better tolerated and results in more effective colon cleansing compared with PEG [8-13]. 
Despite its popularity, $\mathrm{NaP}$ generally fails to show better efficacy than PEG-ELS.

In some less comprehensive studies, PEG-ELS preparation even yields better results [14-17].

The purpose of this prospective randomized observerblinded multicenter study was to compare not only tolerability as the primary aim, but also the efficacy and safety profile of the bowel preparation solely using $\mathrm{NaP}$ versus $\mathrm{NaP}$ and PEG in combination with sennosides as an adjunctive laxative.

\section{PATIENTS AND METHODS}

A total of 360 patients were recruited during a four-month period, of which 355 consecutive outpatients aged between 18 and 75 years were included in the study, undergoing colonoscopy in 5 endoscopy centres after cleansing preparation. Indications for colonoscopy were history or symptoms of intestinal complaints, weight loss, anaemia, or control of persistent diseases. Patients with an urgent indication for colonoscopy, intolerance to one of the used components, or insufficient compliance were excluded from the study. Patients were randomized into three preparation protocols and analysed for tolerability, efficacy, and safety. They were instructed to remain on a clear liquid diet for 24 hours prior to the procedure. According to the type of the study using well-established routine procedures, for legal reasons no formal ethical approval was necessary. The study was conducted in line with the ethical commitments of the hospital. All patients gave written informed consent. The study was sponsored by Ferring, Germany, without any intellectual or substantial input into the study protocol and analysis of results.

\section{BOWEL PREPARATION}

\section{1. $N a P(g r o u p A)$}

$\mathrm{NaP}$ (Fleet Phospho-soda, Ferring, Germany) was administered in two doses of $45 \mathrm{~mL}$ each, containing $10.8 \mathrm{~g}$ disodium phosphate dodecahydrate and $24.4 \mathrm{~g}$ sodium dihydrogen phosphate dihydrate. According to the protocol, the first dose was given in the evening before the examination (19:00 p.m.), and the second in the morning before the procedure (7:00 a.m.). Each dose was diluted in $120 \mathrm{~mL}$ of cold water followed by other $240 \mathrm{~mL}$ of cold water. The day before colonoscopy the patient was encouraged to drink at least $3 \mathrm{~L}$ or more of clear liquids.

\subsection{NaP + sennosides (group B)}

In addition to Group A protocol, sennosides preparation (X-Prep, Mundipharma, Germany) as a supplemental laxative was given the day before the procedure (14:00 p.m.). One dose of $75 \mathrm{~mL}$ contains $150 \mathrm{mg}$ of standardized hydroxyanthracene-glycosides (sennoside B). Laxative effects start 5-8 hours after the intake. In addition, the patients had to drink at least a glass $(240 \mathrm{~mL})$ of cold water. The day before
TABLE 1: Cleanliness quality score of the blinded endoscopist.

\begin{tabular}{ll}
\hline 0 & Dry colon, no solid faeces \\
1 & Only transparent fluid \\
2 & Fluid faeces \\
3 & Small amount of solid faeces, $\geq 90 \%$ of the mucosa visible \\
4 & Solid faeces, $<90 \%$ of the mucosa visible \\
\hline
\end{tabular}

colonoscopy the patient was encouraged to drink at least $3 \mathrm{~L}$ or more of clear liquids within the following 4 hours.

\subsection{PEG-ELS and sennosides (group C)}

Patients receiving PEG-ELS were instructed to remain on clear liquids (3-4L) after a light meal at 13:00 p.m. the day before the examination. One dose of sennosides (XPrep) was administered at 14:00 p.m.. Before 7:00 a.m. on the day of the procedure the patients were to drink 4 sachets of PEG-ELS (Klean-Prep) diluted in $1 \mathrm{~L}$ of water per dose, containing $68.96 \mathrm{~g}$ of PEG 3350 with potassium chloride, sodium chloride, sodium hydrogen carbonate, and sodium sulphate. The intake of PEG-ELS $(250 \mathrm{~mL}$ each within 10-15 minutes) before colonoscopy was continued until cleanliness of faeces was proven or a maximum volume of $4 \mathrm{~L}$ was consumed.

Prior to the examination, patients rated their tolerability of the drinking volume, and the entire preparation received a rating of good, moderate, or poor. Adverse events were recorded according to GCP in detail in the case-report form and carefully monitored by the trial investigator and the sponsor. They were also evaluated (none, mild, moderate, and severe) by each patient. All recorded adverse events were specified and listed in the final report.

Demographic data, comorbidity, and medication of the study population were also documented before allocation to the corresponding preparation regimen according to the random list.

Each colonoscopy was performed by an experienced endoscopist blinded with regard to the preparation protocol. Commencement of the endoscopic procedure was between 11:00 a.m. and 1:00 p.m. The acquisition of information about bowel cleansing methods was not permitted. Premedication, vital signs, and complications during the procedure were documented including reasons for incomplete endoscopy. Discomfort of the ingested fluids was qualified in a verbal rating scale as "no, slight, moderate, or strong discomfort." The quality of cleanliness and visibility of the colonic mucosa were scored according to a modified fivelevel rating scale used in prior clinical studies [7-9, 17-19] (see Table 1).

\subsection{Statistics}

Statistical analysis was based on group randomisation to enable allocation of each consecutive patient to the predetermined random list. Computer-generated randomisation lists were applied to each participating centre. Each working day was assigned to one treatment group, resulting in the same 
preparation protocol for all patients studied at a particular day. Comparison of the three preparation modalities $A, B$, and $\mathrm{C}$ was realized by analysis of variance (ANOVA), $t$ test, U-test, $\chi^{2}$ (chi-square) test for testing each regimen pair, Kruskal-Wallis test (K-W test), Mantel-Haenszel $X^{2}$ test for additional pair comparison $\left(X_{\mathrm{MH}}^{2}\right.$ : test of linear relation between columns of cross-tables), analysis of factors, and test of equivalence for responder rates. All tests were conducted two-sided. $P<.05$ was considered to demonstrate statistical significance or equivalence, and respective numbers were given as mean \pm standard deviation. The primary objective was to demonstrate a $20 \%$ superior tolerability of the treatment arm A. If significant, equivalent efficacy of $\mathrm{NaP}$ + sennosides (B) compared with PEG-ELS + sennosides (C) was tested (good and excellent effects) on the basis of a level of equivalence of $15 \%$ and a success rate of at least $85 \%$ of the standard which was defined hypothetically as PEG-ELS + sennosides. If significant, equivalent efficacy of $\mathrm{NaP}$ (A) compared with PEG-ELS + sennosides (C) was tested on the basis of a level of equivalence of $15 \%$ and a success rate of at least $85 \%$ of the standard. Accordingly, group sizes of at least 107 patients were calculated to test superiority with a power of $1-\beta=0.80$. For data management and statistical analyses, the SAS software was used.

\section{RESULTS}

355 out of 360 patients between 17 and 82 years (median age of 59 years) participated in the study with an indication for a complete colonoscopy. Comorbidity was present in 194 patients $(54.6 \%)$, mostly with cardiovascular diseases (23.1\%) followed by diseases of gastrointestinal tract (16.9\%) and endocrine or metabolic abnormalities (13.0\%). Medication of concomitant diseases was documented in 174 patients (49\%). Characteristics of the patients randomized into the three groups (A, B, and C) are outlined in Table 2 .

The three groups were similar with regard to age, BMI, indication for colonoscopy, and comorbidities. Compliance with the cleansing protocol was also comparable between groups A (99.2\%), B (97.7\%), and C (95.7\%). All patients were examined in 5 centres. The distribution of patients to each centre (C) was as follows: $\mathrm{C} 1: n=73, \mathrm{C} 2: n=69, \mathrm{C} 3$ : $n=36, \mathrm{C} 4: n=111$, and C5: $n=67$. The reason for the disproportion of patients in the groups $\mathrm{A}, \mathrm{B}$, and $\mathrm{C}$ was due to lower numbers of colonoscopies in centres 3 and 4 on the examination days randomized to $\mathrm{C}$.

The total volume of administered fluids for bowel cleansing in the three groups varied (A: $4.33 \mathrm{~L}, \mathrm{~B}: 4.56 \mathrm{~L}$, and C: $4.93 \mathrm{~L}$; ANOVA: $P=.005$, Kruskal-Wallis: $P=.015$ ). Discomfort according to the verbal rating scale showed a significant difference between Group A and Group C in pair analysis (see Figure 1).

All relevant symptoms during preparation were protocolled (nausea, vomiting, abdominal pain or bloating, anal irritation, fatigue, sleep disorder, hunger, weakness, chest pain, chills). Factor analysis showed heterogeneity between the three groups without relevant differences (see Figure 2). Subgroup analyses showed a higher proportion of patients (>70 years) with nausea and vomiting (nausea: 16 versus 9

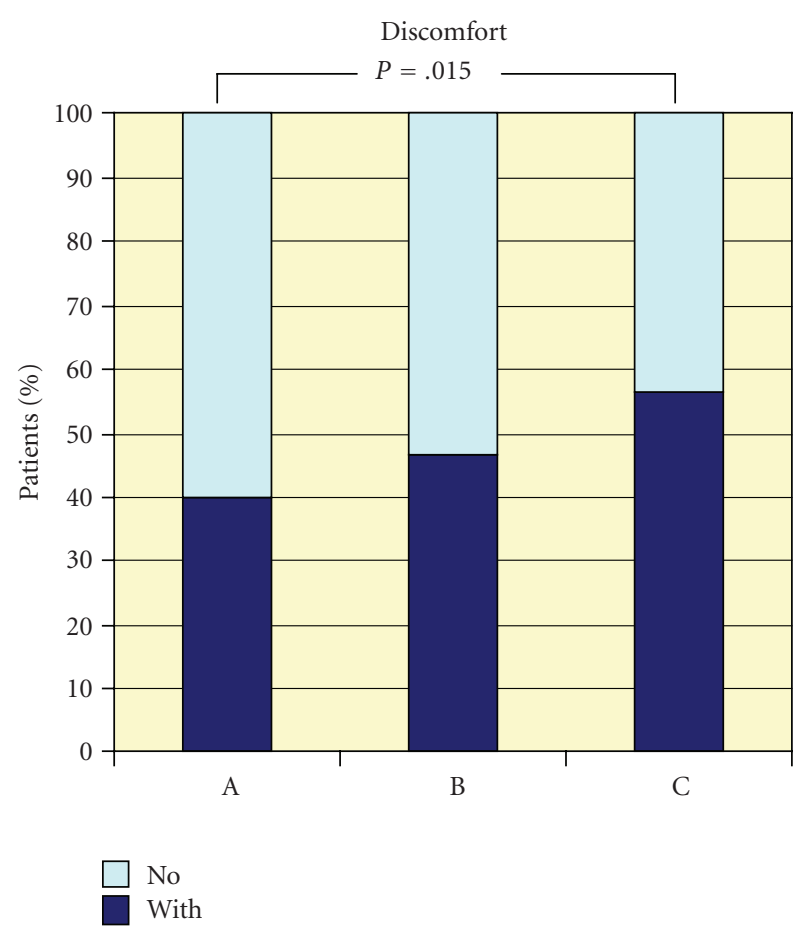

FIgURE 1: Percentages of patients with (slight to strong) or without discomfort of ingested liquids $\left(X^{2}\right.$ test: $\left.P=.015\right)$.
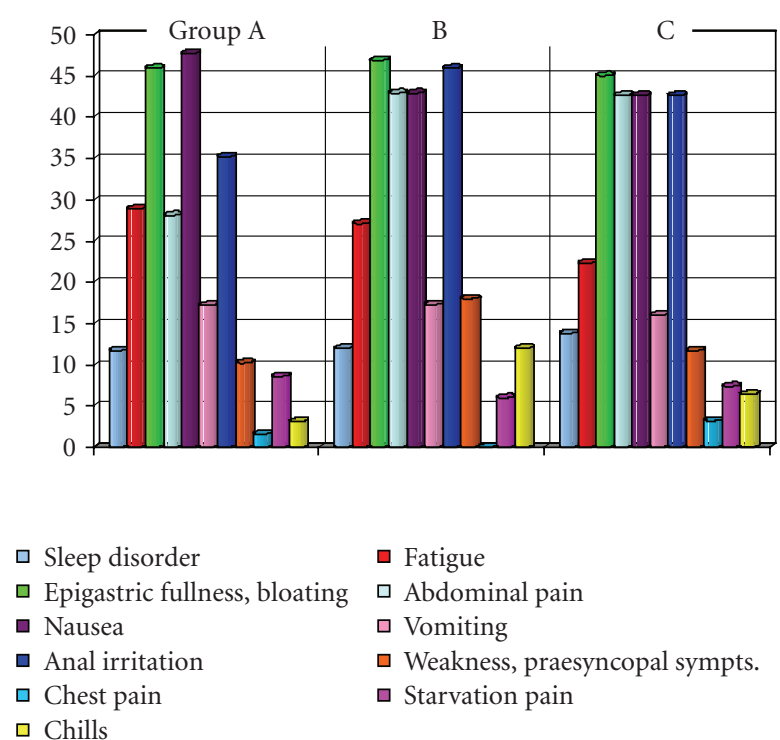

Figure 2: Profile of symptoms during bowel preparation in the three groups (\%).

patients of $>70$ years, 41 versus 67 patients of $<70$ years (KW test: $P=.004)$; vomiting: 9 versus 16 patients of $>70$ years, 14 versus 94 patients of $<70$ years (K-W test: $P=.012)$ ).

No significant difference in global tolerability of each preparation regimen could be ascertained (see Figure 3 ).

Complete examination of the colon was possible in $94.9 \%$ of the patients. Premedication, vital signs, and pain 
TABLE 2: Characteristics of the patients separated by the cleansing protocol (A, B, and C).

\begin{tabular}{|c|c|c|c|c|}
\hline & A & $\mathrm{B}$ & $\mathrm{C}$ & Total \\
\hline Number & 128 & 133 & 94 & 355 \\
\hline Age (median) & 61 & 59 & 57.5 & 59 \\
\hline $\operatorname{BMI}\left(\mathrm{kg} / \mathrm{m}^{2}\right)$ & 25.1 & 24.9 & 24.9 & 25 \\
\hline \multicolumn{5}{|l|}{ Indication for colonoscopy } \\
\hline Abdominal symptoms & $38.3 \%$ & $40.6 \%$ & $38.3 \%$ & $139(39.2 \%)$ \\
\hline Other & $7.8 \%$ & $9.8 \%$ & $7.4 \%$ & $30(8.5 \%)$ \\
\hline Suspected colonic disorder & $23.4 \%$ & $24.1 \%$ & $20.2 \%$ & $90(25.4 \%)$ \\
\hline Control of colonic disease & $32 \%$ & $24.1 \%$ & $20.2 \%$ & $92(25.9 \%)$ \\
\hline Aggravation of colonic disease & $5.5 \%$ & $8.3 \%$ & $8.5 \%$ & $26(7.3 \%)$ \\
\hline Comorbidities & $57.8 \%$ & $49.6 \%$ & $57.4 \%$ & $194(54.6 \%)$ \\
\hline
\end{tabular}

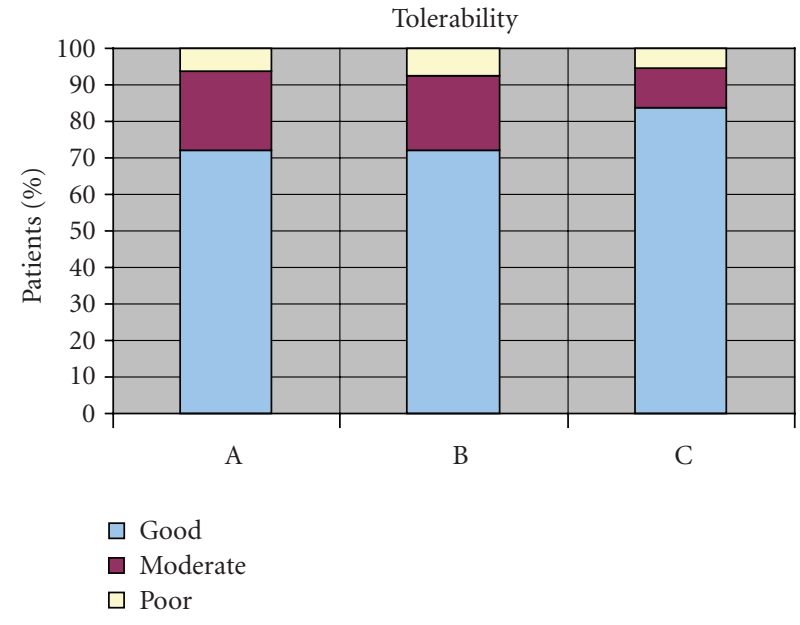

FIGURE 3: Tolerability of the three preparation modalities received a rating of good, moderate, or poor.

before and during colonoscopy were registered and were similar in the three groups. The assessment of the cleanliness quality was evaluable in 337 patients in which complete colonoscopy could be achieved. Cleansing efficacy was high in all three groups, and there was no statistical difference. Group C (PEG-ELS + sennosides) tended towards a better response in the analysis of details with regard to all scores (see Figure 4). The rates of good and very good cleanliness quality were $77.7 \%$ in Group A, $86.7 \%$ in Group B, and $85.2 \%$ in Group C $\left(P=.135\right.$ in $X^{2}$ test) (A versus B: $P=.062, \mathrm{~A}$ versus $\mathrm{C}: P=.171, \mathrm{~B}$ versus $\mathrm{C}: P=.755)$.

Acceptance was high in all groups. Similar proportions of patients were reported to refuse repetition of the same preparation regimen: Group $\mathrm{A}=14.8 \%$, Group $\mathrm{B}=18.5 \%$, and Group $\mathrm{C}=17 \%\left(X^{2}\right.$ test: $\left.P=.737\right)$. If there is alternative preparation offered for colonoscopy, patients would prefer another protocol in A: 30.2\%, B: 30\%, and C: $37.2 \%\left(X^{2}\right.$ test: $P=.445)$.

\section{DISCUSSION}

Colonoscopy is an important tool for the diagnosis and follow-up of colonic disorders and the prevention of neo- plasms. There is an ongoing search for the ideal cleansing preparation, aiming at better patient compliance, shorter colonic preparation time, and better cleansing effects. Consumption of a large amount of fluid is frequently associated with poor patient tolerance. It might therefore be expected that a decrease in volume could lead to a better acceptability.

In the present study, bowel cleansing preparation with $\mathrm{NaP}$ with or without sennosides was compared to a PEG electrolyte solution with sodium sulphate (PEG-ELS) in combination with sennosides in terms of tolerability, patient acceptance, and bowel cleanliness. The investigation included a considerable number of patients. The differences in the sizes of the three treatment arms are due to the randomisation procedure, and may not very likely influence outcomes in the view of very similar baseline characteristics.

Our results of the present study indicate that all three regimens showed a good tolerability with a trend towards PEG-ELS, but this difference did not reach a level of statistical significance. As demonstrated in other studies, $\mathrm{NaP}$ was not better tolerated although the ingested volume of liquids was significantly lower (medium: 14\%) in the NaP group. Subgroup analysis in consideration of age however showed significantly more side effects in patients over 70 years like nausea, vomiting, and abdominal pain in Group B $(\mathrm{NaP}+$ sennosides). Dizziness and presyncope as symptoms caused by volume depletion and hyperphosphatemia were not predominant in the NaP regimens, which is concordant with the literature [20]. Vomiting and abdominal discomfort reported as frequent side effects of PEG-ELS with a better tolerance of $\mathrm{NaP}$ [21] were not increased in the PEG-ELS group.

Acceptance of the three preparation protocols was similar. Less than $20 \%$ of each group would not like to repeat the same regimen for bowel cleansing. Also similar proportions of patients would prefer another cleansing solution for the next colonoscopy with an insignificant trend towards $\mathrm{NaP}$ protocols. Considerably better results in acceptance were reported in literature when $\mathrm{NaP}$ was compared with PEG solutions [8-10, 21-23].

Regarding the cleanliness effects, all three preparation protocols were highly effective with a very good or good response between $77.7 \%$ and $86.7 \%$. Overall cleanliness of the colon was assessed without specifying the cleansing effects in the different bowel segments. According to a 


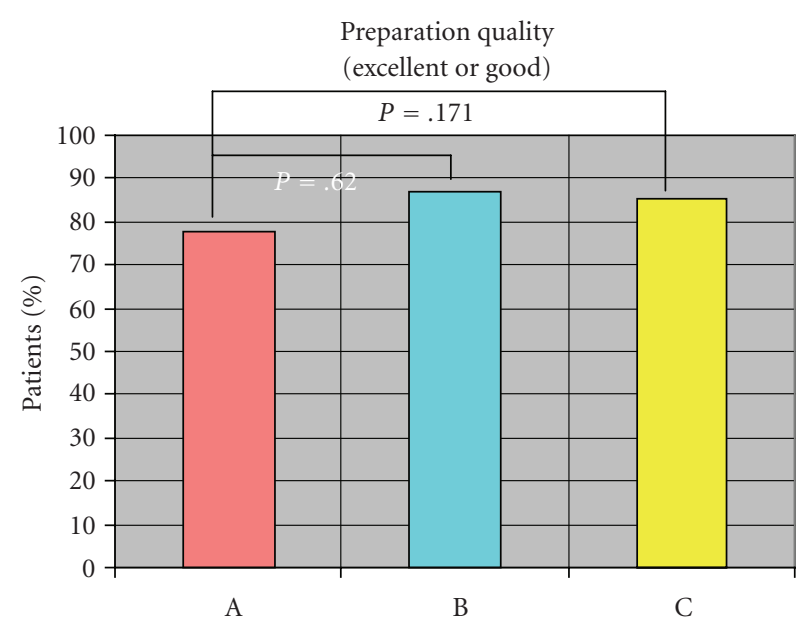

FIgURE 4: Cleanliness responses to bowel preparation as a percentage between the three groups. Cleansing rating includes excellent and $\operatorname{good}(P=.013)$.

worst-case analysis, solid faeces in any of the segments was judged as cleanliness score 4 and transparent fluid in all segments as good (score 1). In contrast, Ell et al. [27] analysed the effects of bowel preparation of different segments, which gives some additional information. Because of the high number of patients, we chose a less complex protocol, also omitting systematic videotaping.

Although there was a trend towards better cleanliness with PEG-ELS plus sennosides, the differences showed no statistical significance. Similar responses were reported by others [18, 22-26].

It is widely established to perform bowel cleansing with PEG-ELS within 2 consecutive days. Because of adding sennosides to the preparation protocol taken the day before the examination, there was no distribution of the amount of PEG-ELS on two days with an overnight pause. Hence, in our protocol the whole PEG-ELS dose was taken over at least 46 hours on the day of the examination under strict control of the unblinded endoscopy assistant nurses of each centre. Then colonoscopy was commenced.

In a recent study by Ell et al. [27], the PEG-ELS (with sodium sulphate) treatment group demonstrated the optimal cleansing quality in all colon segments compared to PEG-ELS (without sodium sulphate) and NaP. No details were given on the amount of liquids taken together with each preparation regimen, which in our opinion plays an important role in the NaP cleansing. Furthermore, most of the examined subjects were inpatients (86\%)-a treatment group with generally more severe illnesses or at least more severe comorbidity which may affect bowel preparation. Therefore, it cannot be compared with our treatment group made up exclusively of outpatients. PEG-ELS doses were administered in two portions on the day before and the day of the examination, which might be an advantage referring to cleanliness of proximal colonic segments [19].

Despite the administration in a two-step procedure with an overnight pause, $\mathrm{NaP}$ showed no better results than PEG-ELS plus sennosides as in the study of Frommer [10].
Colonoscopy was performed at the earliest 3-4 hours after the second dose of $\mathrm{NaP}$.

The combination of $\mathrm{NaP}$ with sennosides, a commonly used laxative to optimize bowel preparation for diagnostic procedures [28], produces a slightly better cleanliness response than $\mathrm{NaP}$ alone. But this minor synergistic effect does not reach statistical significance. On the other hand, there was a tendency towards more side effects, as mentioned above.

A meta-analysis of the trials comparing $\mathrm{NaP}$ with $\mathrm{PEG}$ solutions concluded that oral $\mathrm{NaP}$ was better tolerated by patients and was at least as efficacious [29].

But heterogeneous groups of patients, outpatients, and inpatients and different protocols were mixed up, which limits the conclusion.

Each preparation protocol was carried out according to the dosing instructions recommended for afternoon colonoscopies. Being consistent with the recommendation of the ASGE, patients with severe renal insufficiency or unstable cardiac diseases were excluded from the study [30].

\section{CONCLUSION}

In conclusion, tolerability, acceptance, and the effectiveness of the bowel cleansing regimens for elective colonoscopy with two-dose, low-volume NaP and 4 L PEG-ELS plus sennosides were comparable. Sennosides as adjunctive laxative have only minor synergistic effects when added to $\mathrm{NaP}$, and cannot be recommended for routine endoscopy.

There are still conflicting data regarding the optimal bowel preparation for colonoscopy. This study was carried out to compare the tolerability, safety, and efficacy of three (two) widely used bowel lavage solutions in a large cohort of outpatients. Tolerability and safety were evaluated by means of a symptom questionnaire completed by each patient before the procedure. The scoring of cleanliness was performed during each examination by the blinded endoscopist. The treatment groups were comparable with regard to the baseline characteristics.

\section{ACKNOWLEDGMENTS}

The study was sponsored by Ferring, Germany, without any intellectual or substantial input into the study protocol and analysis of results. The biometrical data were analysed and evaluated by an independent institute of statistics (Institut für Angewandte Statistik, Dr. Jörg Schnitker GmbH, Bielefeld).

\section{REFERENCES}

[1] W. H. Kim, Y. J. Cho, J. Y. Park, P. K. Min, J. K. Kang, and I. S. Park, "Factors affecting insertion time and patient discomfort during colonoscopy," Gastrointestinal Endoscopy, vol. 52, no. 5, pp. 600-605, 2000.

[2] D. K. Rex, T. F. Imperiale, D. R. Latinovich, and L. L. Bratcher, "Impact of bowel preparation on efficiency and cost of colonoscopy," American Journal of Gastroenterology, vol. 97, no. 7, pp. 1696-1700, 2002. 
[3] G. R. Davis, C. A. Santa Ana, S. G. Morawski, and J. S. Fordtran, "Development of a lavage solution associated with minimal water and electrolyte absorption or secretion," Gastroenterology, vol. 78, no. 5, pp. 991-995, 1980.

[4] D. E. Beck, F. J. Hartford, J. A. DiPalma, and C. E. Brady, "Bowel cleansing with polyethylene glycol electrolyte lavage solution," Southern Medical Journal, vol. 78, no. 12, pp. 14141416, 1985.

[5] G. Thomas, S. Brozinsky, and J. I. Isenberg, "Patient acceptance and effectiveness of a balanced lavage solution (Golytely) versus the standard preparation for colonoscopy," Gastroenterology, vol. 82, no. 3, pp. 435-437, 1982.

[6] J. T. DiPiro, K. A. Michael, B. A. Clark, et al., "Absorption of polyethylene glycol after administration of a PEG-electrolyte lavage solution," Clinical Pharmacy, vol. 5, no. 2, pp. 153-155, 1986.

[7] S. J. Vanner, P. H. MacDonald, W. G. Paterson, R. S. A. Prentice, L. R. Da Costa, and I. T. Beck, "A randomized prospective trial comparing oral sodium phosphate with standard polyethylene glycol-based lavage solution (Golytely) in the preparation of patients for colonoscopy," American Journal of Gastroenterology, vol. 85, no. 4, pp. 422-427, 1990.

[8] B. E. Kolts, W. E. Lyles, S. R. Achem, L. Burton, A. J. Geller, and T. MacMath, "A comparison of the effectiveness and patient tolerance of oral sodium phosphate, castor oil, and standard electrolyte lavage for colonoscopy or sigmoidoscopy preparation," American Journal of Gastroenterology, vol. 88, no. 8, pp. 1218-1223, 1993.

[9] S. M. Cohen, S. D. Wexner, S. R. Binderow, et al., "Prospective, randomized, endoscopic-blinded trial comparing precolonoscopy bowel cleansing methods," Diseases of the Colon and Rectum, vol. 37, no. 7, pp. 689-696, 1994.

[10] D. Frommer, "Cleansing ability and tolerance of three bowel preparations for colonoscopy," Diseases of the Colon and Rectum, vol. 40, no. 1, pp. 100-104, 1997.

[11] A. P. Chilton, M. O’Sullivan, M. A. Cox, D. E. Loft, and C. U. Nwokolo, "A blinded, randomized comparison of a novel, lowdose, triple regimen with fleet phospho-soda: a study of colon cleanliness, speed and success of colonoscopy," Endoscopy, vol. 32, no. 1, pp. 37-41, 2000.

[12] A. Arezzo, "Prospective randomized trial comparing bowel cleaning preparations for colonoscopy," Surgical Laparoscopy, Endoscopy and Percutaneous Techniques, vol. 10, no. 4, pp. 215$217,2000$.

[13] C. J. Young, R. R. Simpson, D. W. King, and D. Z. Lubowski, "Oral sodium phosphate solution is a superior colonoscopy preparation to polyethylene glycol with bisacodyl," Diseases of the Colon and Rectum, vol. 43, no. 11, pp. 1568-1571, 2000.

[14] P. Pontone, G. Bogliolo, V. Pietropaolo, and P. Pallotto, "Polyethylene glycol versus sodium phosphate: comparison of 2 preparations for colonoscopy," Il Giornale di Chirurgia, vol. 17, no. 3, pp. 128-131, 1996.

[15] J. B. Marshall, J. S. Barthel, and P. D. King, "Short report: prospective, randomized trial comparing a single dose sodium phosphate regimen with PEG-electrolyte lavage for colonoscopy preparation," Alimentary Pharmacology and Therapeutics, vol. 7, no. 6, pp. 679-682, 1993.

[16] M. G. Lapalus, J. L. Gaudin, T. Lemkecher, et al., "Prospective randomized single-blind trial comparing oral sodium phosphate with polyethylene glycol for colonoscopy preparation," Gastroenterologie Clinique et Biologique, vol. 25, no. 1, pp. 2934, 2001.

[17] A. P. Chilton, M. O'Sullivan, M. A. Cox, D. E. Loft, and C. U. Nwokolo, "A blinded, randomized comparison of a novel, low- dose, triple regimen with fleet phospho-soda: a study of colon cleanliness, speed and success of colonoscopy," Endoscopy, vol. 32, no. 1, pp. 37-41, 2000.

[18] W. K. Clarkston, T. N. Tsen, D. F. Dies, C. L. Schratz, S. K. Vaswani, and P. Bjerregaard, "Oral sodium phosphate versus sulfate-free polyethylene glycol electrolyte lavage solution in outpatient preparation for colonoscopy: a prospective comparison," Gastrointestinal Endoscopy, vol. 43, no. 1, pp. 4248, 1996.

[19] A. M. A. El Sayed, Z. A. Kanafani, F. H. Mourad, et al., "A randomized single-blind trial of whole versus splitdose polyethylene glycol-electrolyte solution for colonoscopy preparation," Gastrointestinal Endoscopy, vol. 58, no. 1, pp. 3640, 2003.

[20] L. C. Hookey, W. T. Depew, and S. Vanner, "The safety profile of oral sodium phosphate for colonic cleansing before colonoscopy in adults," Gastrointestinal Endoscopy, vol. 56, no. 6, pp. 895-902, 2002.

[21] L. Bujanda, C. Muñoz, A. Sánchez, et al., "Tolerance to and colon cleansing with 2 preparations. Polyethylene glycol or sodium phosphate," Gastroenterología y Hepatología, vol. 24, no. 1, pp. 9-12, 2001.

[22] J. B. Marshall, J. J. Pineda, J. S. Barthel, and P. D. King, "Prospective, randomized trial comparing sodium phosphate solution with polyethylene glycol-electrolyte lavage for colonoscopy preparation," Gastrointestinal Endoscopy, vol. 39, no. 5, pp. 631-634, 1993.

[23] S. A. Afridi, J. S. Barthel, P. D. King, J. J. Pineda, and J. B. Marshall, "Prospective, randomized trial comparing a new sodium phosphate-bisacodyl regimen with conventional PEGES lavage for outpatient colonoscopy preparation," Gastrointestinal Endoscopy, vol. 41, no. 5, pp. 485-489, 1995.

[24] A. Thomson, P. Naidoo, and B. Crotty, "Bowel preparation for colonoscopy: a randomized prospective trial comparing sodium phosphate and polyethylene glycol in a predominantly elderly population," Journal of Gastroenterology and Hepatology, vol. 11, no. 2, pp. 103-107, 1996.

[25] J. Lee, K. McCallion, A. G. Acheson, and S. T. Irwin, "A prospective randomised study comparing polyethylene glycol and sodium phosphate bowel cleansing solutions for colonoscopy," Ulster Medical Journal, vol. 68, no. 2, pp. 68-72, 1999.

[26] C. M. Poon, D. W. H. Lee, S. K. Mak, et al., "Two liters of polyethylene glycol-electrolyte lavage solution versus sodium phosphate as bowel cleansing regimen for colonoscopy: a prospective randomized controlled trial," Endoscopy, vol. 34, no. 7, pp. 560-563, 2002.

[27] C. Ell, W. Fischbach, R. Keller, et al., "A randomized, blinded, prospective trial to compare the safety and efficacy of three bowel-cleansing solutions for colonoscopy," Endoscopy, vol. 35, no. 4, pp. 300-304, 2003.

[28] B. A. van Gorkom, A. Karrenbeld, A. J. Limburg, and J. H. Kleibeuker, "The effect of sennosides on colonic mucosal histology and bowel preparation," Zeitschrift für Gastroenterologie, vol. 36, no. 1, pp. 13-18, 1998.

[29] C.-W. Hsu and T. F. Imperiale, "Meta-analysis and cost comparison of polyethylene glycol lavage versus sodium phosphate for colonoscopy preparation," Gastrointestinal Endoscopy, vol. 48, no. 3, pp. 276-282, 1998.

[30] American Society for Gastrointestinal Endoscopy, "Preparation of patients for gastrointestinal endoscopy," Gastrointestinal Endoscopy, vol. 48, no. 6, pp. 691-694, 1998. 


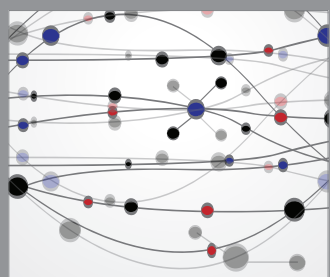

The Scientific World Journal
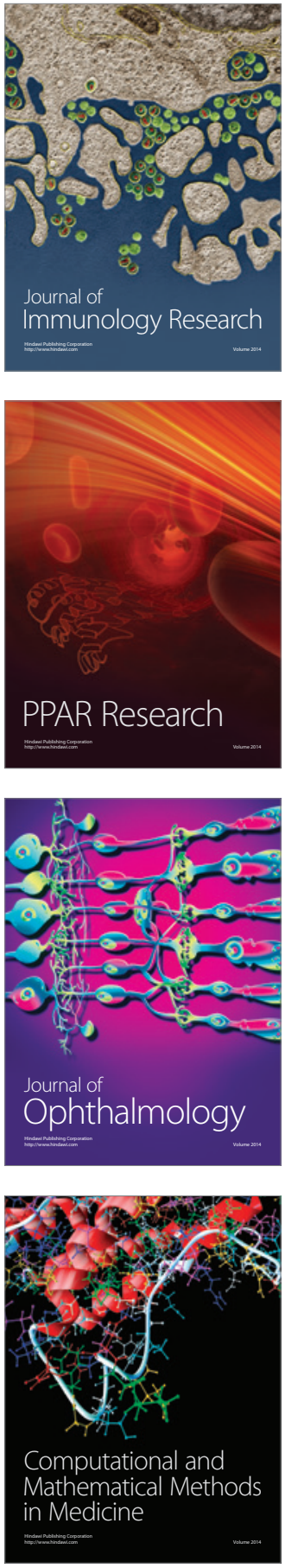

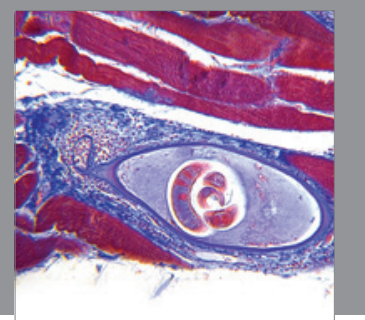

Gastroenterology

Research and Practice
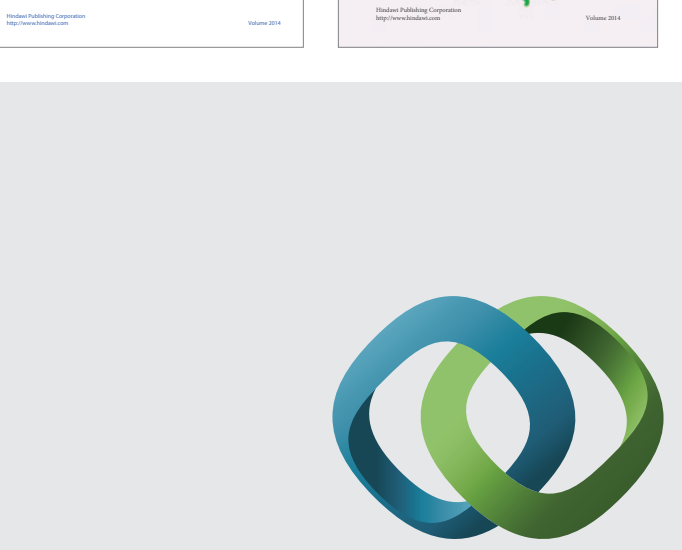

\section{Hindawi}

Submit your manuscripts at

http://www.hindawi.com
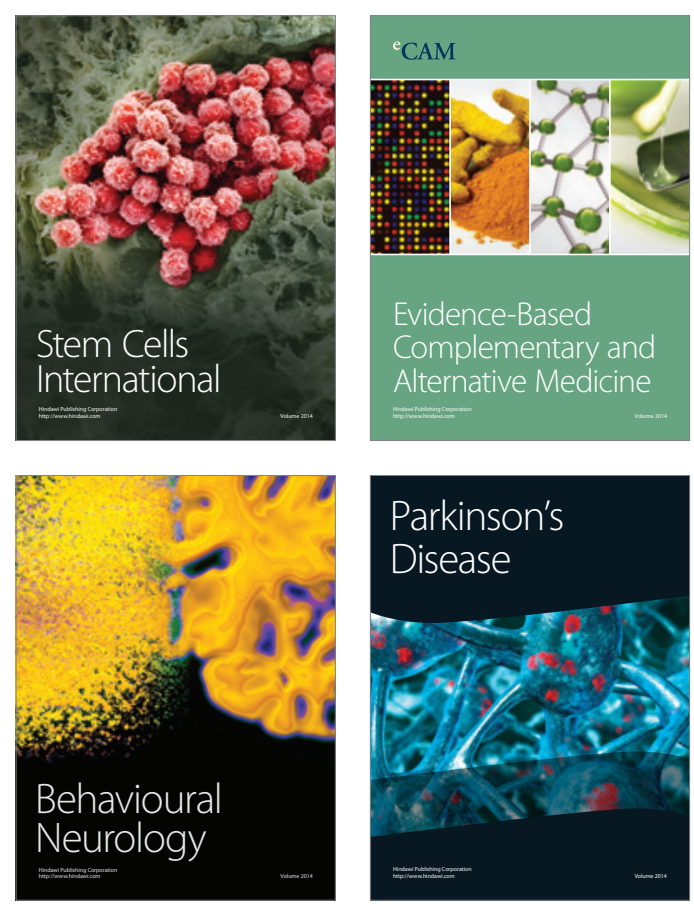

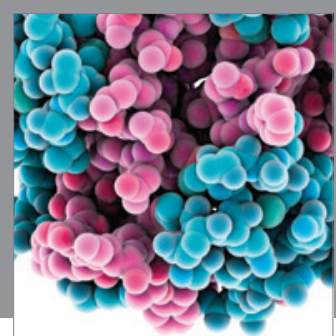

Journal of
Diabetes Research

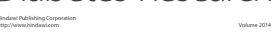

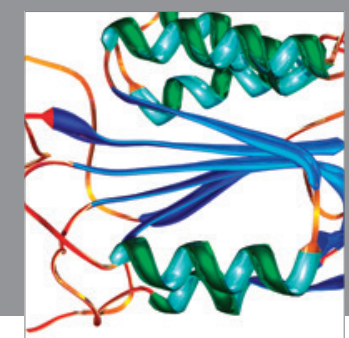

Disease Markers
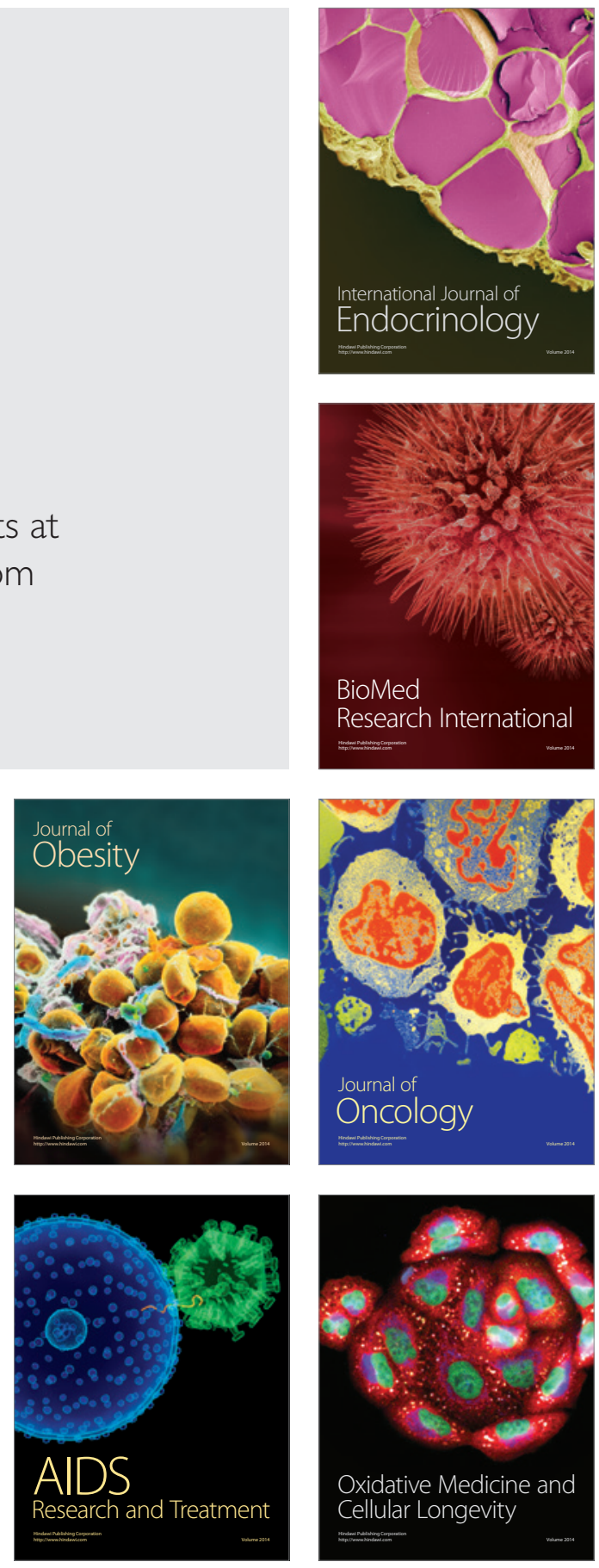